Introduction Occupational exposure to dust, gases and fumes has been associated with chronic airway disease or poor lung function in several workforce based studies. However, workforce studies may underestimate such associations because of healthy worker effect confounding bias. We conducted a community-based study that used a more generalised populations of individuals and less susceptible to healthy worker effect. We investigated the associations between the self-reported occupational exposure and chronic bronchitis, as well as pulmonary function testing.

Methods The study population is the Third-Generation cohort from Framingham Heart Study with a total of 3,894 participants. We used participants' examination of FEV1, FEV1/FVC, chronic bronchitis (based on self reported symptoms) as outcomes. Occupational exposure was assessed by self-reported exposure to vapours, gas, dust, or fumes at work. Gender, age, height, pack-years, and smoking status were used as covariates in our analysis. We used linear mixed effect models for continuous outcomes and generalised estimating equations for dichotomous outcomes due to the family structure.

Results There are 1,745 participants reporting occupational exposure at work and 2,149 participants reporting no occupational exposure. The association of occupational exposure on the $\mathrm{FEV}_{1}$ and $\mathrm{FEV}_{1} / \mathrm{FVC}$ was not significant in this cohort. However, self-reported occupational exposure was associated with a risk of chronic bronchitis after controlling for covariates (OR $1.55,95 \%$ CI 1.19 to 2.01 ). Current smoking was associated with a greater risk of chronic bronchitis after controlling for covariates (OR 3.20, 95\% CI 2.37 to 4.32). Those with combined occupational and smoking exposure had a 5 fold increased risk of chronic bronchitis compared those with neither occupational nor smoking exposure.

Conclusions Occupational exposure is significantly associated with chronic bronchitis. In addition to preventing smoking exposure, preventive strategies should be taken by clinicians and health policy-makers to reduce occupational exposures in workplace.

\section{S133 COPD, OCCUPATION AND QUALITY OF LIFE AMONG RESIDENTS OF A HISTORICALLY INDUSTRIALISED AREA}

doi:10.1136/thoraxjnl-2012-202678.138

LGB Lewis, A Darby, J Waterhouse, D Fishwick. Sheffield Teaching Hospitals, Sheffield, United Kingdom

Introduction COPD is known to significantly affect health and quality of life, and is increasingly recognised to have a significant contribution from occupational exposures, particularly vapours, gases, dusts and fumes (VGDF). However, there is a paucity of evidence exploring the relationship between exposure to VGDF at work and health-related quality of life.

Methods A random selection of adults aged over 55 years in the Sheffield area of the UK were mailed a self-completed questionnaire (including questions on respiratory symptoms and physiciandiagnosed disease, smoking and occupational history); responders were invited to perform lung function ( $\mathrm{FEV}_{1}$ and $\left.\mathrm{FVC}\right)$, and to complete the EQ-5D-3L quality of life instrument. A measure of socioeconomic deprivation (SED), using the proportion of individuals within a participants postal code receiving income support, was also collected. Results Out of 4000 questionnaires, 2001 were returned. From these, 623 provided further data as detailed above. $57 \%$ were male, $62 \%$ had been, or were, smokers, $24 \%$ had a physician diagnosis of COPD and $62 \%$ reported having been exposed to VGDF in the past. In univariate analysis those with COPD were more likely to be older, have smoked, been exposed to VGDF and have a lower quality of life (all $p<0.001)$. A history of exposure to VGDF was associated with a lower quality of life $(p<0.001)$. Both VGDF exposure and COPD were associated with greater levels of SED $(p<0.001)$. A linear regression analysis was performed using the EQ_5D summary index as the dependant variable and age, gender, SED, smoking status, physician diagnosed COPD and percentage predicted $\mathrm{FEV}_{1}$ and
VGDF exposure as independent variables. Female gender, greater SED, VGDF exposure and physician diagnosed COPD were identified as predictors of reduced quality of life (as measured by EQ-5D VAS and summary index scores).

Discussion These results support the link between COPD and reduced quality of life, and additionally provide evidence to link occupational exposures to VGDF to a reduction in quality of life. These findings are of significance to health care professionals and policy makers, given future expectations for longer working lives.

\section{S134 COMPARISON OF SPECIFIC INHALATION CHALLENGE (SIC) WITH OASYS ANALYSIS OF SERIAL PEF ANALYSIS IN THE DIAGNOSIS OF OCCUPATIONAL ASTHMA}

doi:10.1136/thoraxjnl-2012-202678.139

VC Moore, PS Burge. Birmingham Heartlands Hospital, Birmingham, England

Aims to compare Specific inhalation challeng (SIC) with serial measurements of PEF in the diagnosis of occupational asthma Methods; All workers having SIC with occupational agents over a 3 year period were included. Their serial PEF records made during exposure to the suspected agents where analysed using Oasys software. Positive records were those with any of the following; Oasys score $>2.5 ; \mathrm{ABC}$ score $\geq 15$ litres $/ \mathrm{min} / \mathrm{hr}$ or timepoint $\geq 1$ non-waking reading Results; 211 challenges were done in 51 workers. 45/51 kept serial PEF records suitable for Oasys analysis. SIC and Oasys analysis were concordant in $17 / 45$ (38\%), particularly those exposed to isocyanates or metal-working fluids. SIC was positive in 5 workers with equivocal Oasys analysis in line with its known sensitivity of c70-80\%. 12 workers had negative or non-asthmatic SIC responses with positive Oasys analysis. Further investigation showed that occupational asthma was the most likely diagnosis. Negative SIC responses were due failure to identify the correct causative agent or problems with reproducing the work exposures. This was a particular problem with cleaning agents where a protein source may be needed to convert chlorine-releasing agents to chloramines (as shown in swimming pool asthma). Nine workers had equivocal challenges and clearly positive Oasys analysis, helping to clarify the diagnosis in this group, again non-standard agents were common in this group.

Conclusion SIC and serial PEF analysis are complementary methods for validating a diagnosis of occupational asthma. SIC has particular problems when methods of exposure for newer agents have not been fully developed, Oasys analysis lacks sensitivity when current specificity is fixed at $>90 \%$.

Abstract $\$ 134$ Table 1

\begin{tabular}{lccc}
\hline & \multicolumn{3}{c}{ Oasys } \\
\cline { 2 - 4 } SIC & Positive & Equivocal & Negative \\
\hline Positive & 16 & 5 & 0 \\
Equivocal & 9 & 1 & 1 \\
Rhinitis etc & 5 & 0 & 0 \\
Negative & 7 & 1 & 1 \\
\hline
\end{tabular}

\section{S135}

ASTHMA, OCCUPATION AND QUALITY OF LIFE IN A HISTORICALLY INDUSTRIALISED AREA OF THE UNITED KINGDOM

doi:10.1136/thoraxjnl-2012-202678.140

LGB Lewis, A Darby, J Waterhouse, D Fishwick. Sheffield Teaching Hospitals, Sheffield, United Kingdom

Introduction Whilst harmful inhaled workplace exposures are known to be associated with either the development or aggravation 